\title{
Anatomy of lumbar facet joint: a comprehensive review
}

\author{
S. Kapetanakis ${ }^{1,2}$, N. Gkantsinikoudis ${ }^{1}$ \\ ${ }^{1}$ Spine Department and Deformities, Interbalkan European Medical Centre, Thessaloniki, Greece \\ 2Department of Minimally Invasive and Endoscopic Spine Surgery, Athens Medical Centre, Athens, Greece
}

[Received: 30 July 2020; Accepted: 4 October 2020; Early publication date: 12 October 2020]

\begin{abstract}
Lumbar facet joints (LFJs) are diarthrodial joints which provide articulation between two adjacent lumbar vertebrae. LFJs represent complex anatomic structures with multifaceted biomechanical and functional characteristics. They are theorized as structures of crucial clinical significance since their degenerative morphologic alterations are frequently related to emergence of low back pain. Despite the emerging interest in describing LFJs anatomy in recent years, precise description of LFJs innervation remains controversial. In this comprehensive review, anatomy and biomechanical importance of LFJs and associated adjacent extra-articular structures are thoroughly presented. Furthermore, LFJs innervation in respect to current literature data is punctually analysed. Knowledge of anatomy and innervation LFJs of critical importance for clinicians and spine surgeons, so that patients are properly evaluated and related therapeutic procedures are rationally performed. (Folia Morphol 2021; 80, 4: 799-805)
\end{abstract}

Key words: lumbar vertebrae, zygapophyseal joints, mammillo-accessory ligament, biomechanics, facet joint tropism, dorsal ramus, medial branch

\section{INTRODUCTION}

Facet joints (FJ), which are also classically described as apophyseal or zygapophyseal joints, represent the only synovial joints of spine $[16,26]$. They are paired diarthrodial joints, posterolaterally articulating the posterior arch between adjacent vertebral levels [26]. Lumbar facet joints (LFJs) constitute primary stabilisers of vertebral column, enabling alongside movements as extension, flexion and rotation $[4,31]$.

The aim of this review article is to describe the precise anatomy of LFJs according to contemporary literature data. Particular emphasis is given to innervation of LFJs.

\section{ANATOMY OF ARTICULAR AND EXTRA-ARTICULAR ELEMENTS}

Articular processes and cartilage

Lumbar facet joints are comprised by the articulation of superior and underlying adjacent vertebra via the paired inferior and superior articular processes, respectively $[10,16]$. The major superior and the minor inferior articular processes (SAP and IAP) represent bony protuberances, emerging vertically from the coalescence of pedicles and laminae of respective vertebral arch, posteriorly to the ipsilateral transverse process $[16,26]$. SAP and IAP are lined with articular hyaline cartilage over the subchondral

Address for correspondence: S. Kapetanakis, MD, PhD, Orthopaedic Surgeon-Spine Surgeon, Spine Department and Deformities, Interbalkan European Medical Centre, Thessaloniki, Greece, Athens Medical Centre, Athens, Greece, tel: +306972707384, fax: +302541067200, e-mail: stkapetanakis@yahoo.gr

This article is available in open access under Creative Common Attribution-Non-Commercial-No Derivatives 4.0 International (CC BY-NC-ND 4.0) license, allowing to download articles and share them with others as long as they credit the authors and the publisher, but without permission to change them in any way or use them commercially. 
bone [26]. Hyaline cartilage of LFJs is characterised by poor vascularisation and cellular infiltration, featuring a considerable healing inability after traumatisation [24]. Articular surfaces of SAP and IAP feature a consistently different morphological pattern. Hence, SAP are characterised by a more concave articular surface, whereas IAP by a more convex one $[16,24]$. In addition, orientation of SAP and IAP in sagittal and coronal planes features a noteworthy differentiation. IAP of superior vertebral level faces an anterior and lateral direction, whereas SAP lies posteriorly, facing medially [26].

\section{LFJs articular cavity}

Lumbar facet joints cavity may be anatomically divided in the $\mathrm{FJ}$ articular space and the $\mathrm{FJ}$ recesses, featuring a capacity of $1-2 \mathrm{~mL}[26,36]$. FJ space represents the anatomic space between the articular cartilage of articulating facets [36]. In contrast, FJ recesses are formed by the redundant encapsulation of LFJ by capsular ligament at the superior and inferior parts of the joint, containing adipose tissue or minor synovial villi [10]. Thorpe Lowis et al. [36] studied 19 cadaveric specimens in order to determine the precise anatomic characteristics of FJ recesses in various spinal regions. It was concluded that FJ recesses presented specific characteristics in respect to particular intervertebral levels. LFJs recesses were equally large and anteromedially and posteromedially located. The anteromedial recess was encountered superiorly, emerging over the upper end of SAP of underlying vertebra. In contrast, posteromedial recess surrounded the lower edge of IAP of supernatant vertebra. LFJs cavity featured no direct communication with the retrodural space, demonstrating an anatomically clear delimitation [36]. LFJ cavity and recesses are detectable in radiologic evaluation with magnetic resonance imaging (Fig. 1).

\section{LFJs capsular ligament}

Lumbar facet joints are, similarly to other synovial diarthrodial joints, completely encapsulated by a capsular ligament (LFCL). LFCL is histologically composed by two distinct layers: an outer layer of parallel with lateral-medial direction and densely organized collagen bundles, and an inner layer of elastic fibres with inconsistent orientation $[5,16]$. Furthermore, LFCL features rich innervation with autonomic and nociceptive nerve fibres, which may reproduce pain in cases of inflammatory or mechanical irritation $[26,31]$.

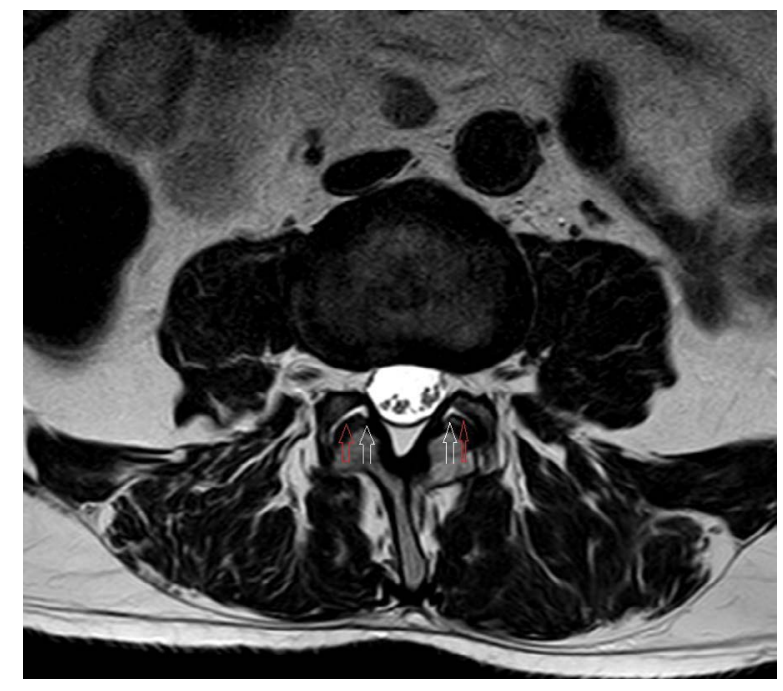

Figure 1. Representation of facet joint cavity (red arrow) and the anteromedially located superior recess (white arrow) of L3-L4 facet joint in a normal lumbar magnetic resonance imaging.

Lumbar facet joints capsular ligament has an important role in maintaining the stability of LFJs. The presence of collagen and elastin administrates substantial mechanical support against shear and tensile forces developed during motion and vertebral loading. LFCL bears a remarkable biomechanical role during various movements of LFJs. It is extended during LFJs lateral bending or rotation. Hence, lateral-medial orientated capsular fibres feature extension along LFJs direction, providing functional resistance and stability. On the other hand, flexion or extension of LFJs is associated with emergence of crucial shear stress, transverse to LFJs direction. In this dynamic condition, LFCL fibres feature also a shear stress transverse to their alignment, providing great resistance [5].

\section{Mammillo-accessory ligament}

Mammillo-accessory ligament (MAL) represents a ligamentous structure bridging the bony mammillary (MP) and accessory processes (AP) of the lumbar vertebra bilaterally. MP constitutes a circular bony protuberance located at the posterior border of SAP. $A P$ is, on the other side, a relatively lesser and sharpening bony structure encountered at the postero-inferior portion of each transverse process root. Ipsilateral MP and AP are connected by the ligamentous MAL (Fig. 2) [12]. MAL constitutes a portion of the medial aspect of intertransverse ligament, featuring a noteworthy tendency to ossification [31].

Shuang et al. [31] dissected 12 cadaveric specimens, in order to determine the precise anatomy of 


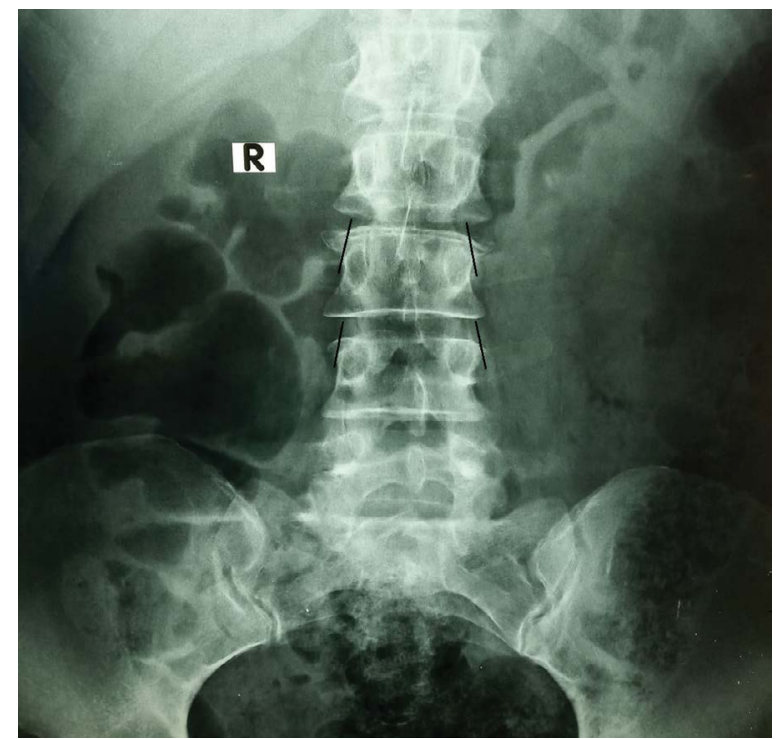

Figure 2. Conceivable representation of mammillo-accessory ligament in an anteroposterior lumbar spine radiograph.

medial branch of spinal dorsal ramus. It was inter alia concluded that the abovementioned anatomic structures form a true fibro-osseous canal, which is anatomically characterized by 4 distinct walls: a superior (MP), an inferior (AP), an anterior (the bony groove between MP and $\mathrm{AP}$ ) and a posterior (MAL). The fibro-osseous canal displays an oblique direction, encountered at the dorsal portion of SAP and origin of transverse process [31]. In the case of MAL ossification, this canal becomes completely osseous [12, 31].

\section{BIOMECHANICAL DATA}

The three-dimensional LFJs and the anteriorly located intervertebral disc at each lumbar spine level compose an anatomic entity described as spinal segment, "three-joint complex" or articular triad [15, $16,21,23,26,32,38]$. These articular structures are theorised to constitute a single unit since the emergence of degenerative alterations in one joint has a subsequent influence on the biomechanical behaviour of the whole unit [26]. Furthermore, the harmonised function of these structures prevents potentially injurious dynamic states, warranting alongside physiological motional activity.

Functionally, the intervertebral disc is majorly responsible for the transmission of axial-compressive forces [16]. In contrast, osseous LFJs are primarily charged with the stabilization of spinal motion segment $[2,3,16]$. However, many biomechanical studies have demonstrated that LFJs also contribute to transfer of implemented axial compressive load on the spine [1, 24, 26, 28, 32]. LFJs may bear up to $25 \%$ of this load, depending on the motion status at each case $[24,41]$. However, the underlying mechanisms of this transmission remain unclear. The nearly vertical inclination of LFJs articular surfaces in conjunction with the existent low friction considerably complicates this description. Inoue et al. [16] reviewed existing literature data on the biomechanical behaviour of LFJs in various dynamic conditions. It was concluded that LFJs may contribute to axial compressive load transfer by three potential mechanisms: by articular surfaces, by LFCL and by the direct connection between the vertebral arch or the pars interarticularis and the tips of articular processes [16].

As stated above, LFJs have been delineated as the primordial lumbar spine stabilizers [22, 32]. LFJs stabilize the respective motion segment in extension and flexion, restricting also axial rotation [26, 39]. More specifically, medial and posterior portion of LFJs instates the major resistance to antero-olisthesis [3]. Comparatively, anatomic construction of LFJs relatively allows flexion-extension motions in sagittal planes, but noteworthy limits axial rotation, so that rotatory instability is prevented [32,39].

Nevertheless, this motion-restrictive pattern may vary according to each lumbar level. This variation is majorly attributed to the differentiated LFJs orientation. LFJs are oriented $82-86$ degrees in regards to the axial plane and 15-70 degrees in regards to the sagittal [5]. Nonetheless, LFJs orientation features a gradual coronal to sagittal conversion from proximal to distal levels [3]. At L1-L2 spinal motion segment, the angle between articular surfaces of SAPs is 30 degrees. However, this angle features a considerable distribution from 30 to 90 degrees in distal (L4-S1) segments. This peculiarity of LFJs orientation in distal segments is profoundly responsible for the emergence of lower resistance to rotational motion [3, 22]. Particular anatomic studies have depicted that the orientation of LFJs gradually approaches the sagittal plane with age [19]. However, the precise contribution of this alteration to further degenerative damage of spinal segment is not adequately understood [18].

\section{LUMBAR FACET JOINTS TROPISM}

Lumbar facet joints tropism (LFJT) is defined as the existing asymmetry between right and left LFJs angle $[8,13,17,37,40]$. Bogduk [6] initially described LFJT as the status where LFJs feature a rotational incongru- 
ity in respect to axial plane, resulting in subsequent asymmetry. More recent studies identified that LFJT may be present in all planes. Therefore, LFJT may also be theorised as the subsistence of asymmetry between left and right LFJs angles in sagittal or coronal planes as well $[3,26]$. This asymmetry is in the majority of cases relatively negligible, under 5 degrees in range. There is currently no universal consensus about the consideration of clinically significant LFJT. Hence, LFJs angulation may normally vary from 5 to 10 degrees [3].

Mohanty et al. [23] performed a retrospective cross-sectional study, in order to elucidate the prevalence of LFJT. For this purpose, 566 intact spinal motion segments from 124 computed tomography scans of spinal trauma patients were analysed. Results showed that LFJT featured greater prevalence in L4-L5 and L5-S1 levels, with the percentages of $47.82 \%$ and $38.5 \%$, respectively. Authors concluded that these results may offer an explanation for the more frequent occurrence of lumbar disc herniation and related degenerative disorders as lumbar arthrosis and degenerative spondylolisthesis encountered at these levels [23]. This thesis has been also adopted from other studies in recent literature $[3,14,26]$.

\section{LUMBAR FACET JOINTS INNERVATION}

Lumbar facet joints innervation is derived by the medial branch of the lumbar dorsal rami, or, as also known, posterior branches of the lumbar spinal nerves [31]. For many years, clinical importance of these structures was not adequately recognized. Hence, precise description of their anatomy was not present even in well-established anatomy atlases [19]. Bogduk et al. [7] were the first to provide a thorough analysis of posterior branches of lumbar spinal nerves anatomy in 1982.

The spinal nerve is divided into four distinct branches, after its exit from the respective intervertebral foramen; the communicating branch, the meningeal branch, the ventral ramus and the smaller dorsal ramus (DR) [31]. At levels L1-L4, DR is separated from the spinal nerve at an approximately right angle $[19,29]$. They subsequently traverse the vertebral foramen, featuring then a dorsal and caudal course $[7,9,30]$. DR is from that point encountered at the orifice formed by the superior border of the adjacent transverse processes and the inferior border of the respective LFJ $[19,20]$. This final aspect of $D R$

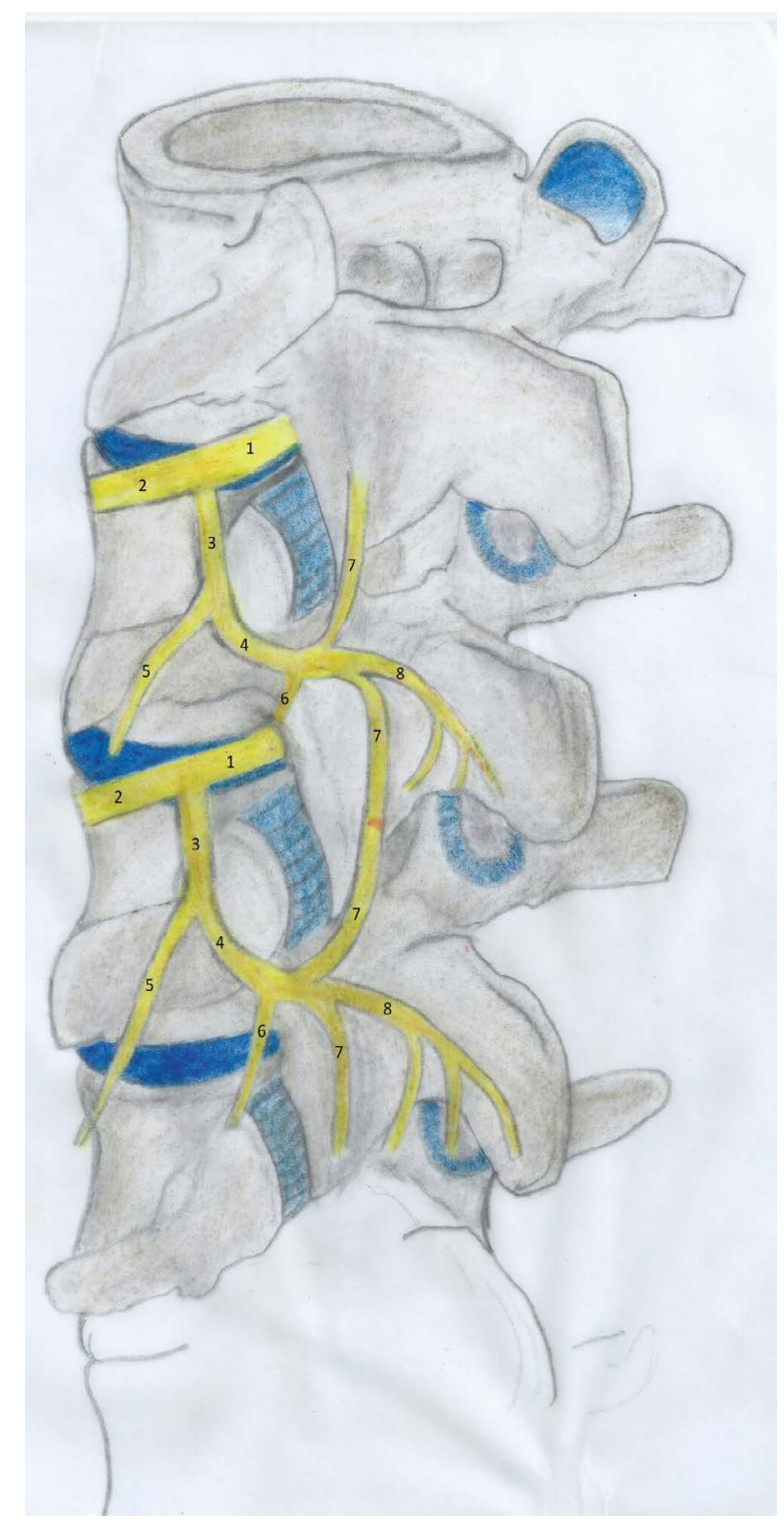

Figure 3. Schematic representation of lumbar dorsal ramus and its branches; 1 - lumbar spinal nerve; 2 - ventral ramus, 3 - dorsal ramus; 4 - medial branch of dorsal ramus; 5 - lateral branch of dorsal ramus; 6 - muscular branch of medial branch of dorsal ramus; 7 - articular branches of medial branch of dorsal ramus; 8 - cutaneous branches of medial branch of dorsal ramus.

is located at the medial portion of intertransverse musculature (Fig. 3) [31].

Dorsal ramus bifurcates, circa $5-10 \mathrm{~mm}$ distal to the abovementioned orifice, into a medial and a lateral branch, forming a 30-degree angle [19, 20]. This division is encountered at the superior edge of the underlying transverse process. There are multiple communicating branches between the adjacent lateral branches, medial branches and dorsal rami [31]. Nerve fibres composition for both branches is duplex, containing sensory as well as motor fibres. Ramifica- 
tion of DR presents also a regional significance; lateral branch innervates tissues lateral to LFJs whereas medial provides innervations to the structures located medially to LFJs line [33] (Fig. 3).

Bogduk et al. [7] proposed that ramification of DR was triple, describing also a distinct intermediate branch in addition to medial and lateral. This thesis was later adopted from other authors as well. Middle or intermediate branch may co-exist with lateral branch in a short common trunk or separate directly from the DR. It is theorized that middle branch provides innervation to the longissimus lumbar muscle [19]. Furthermore, middle branch may also feature rich anastomoses with the other branches, thereby composing complex neural plexuses. Despite the published data about the anatomy of intermediate branch, dichotomy of DR into medial and lateral branches represents the majorly acceptable standard in the literature [31].

After its emergence, medial branch traverses the superior border of the underlying transverse process, proximal to its origin [27]. It then passes between the bases of SAP and adjacent transverse process, traversing the dorsal portion of intertransverse ligament. Medial branch is at this locus accompanied by the posterior branch of the lumbar artery, adhering to the adjacent periosteum via connective tissue [19]. Farther on, it features a medial course passing under the MAL into the fibro-osseous canal. Medial branches of L1-L4 dorsal rami are encountered at the posterolateral portion of this canal, featuring greater proximity to AP than the MP [11]. They subsequently follow a medial and caudal route towards the vertebral lamina, demonstrating thereafter a deep course into the adipose tissue of multifidus muscle [19]. Medial branch is there ramified into three distinct branches; muscular, articular and cutaneous branch, supplying LFJs, multifidus muscles and supra- and inter-spinous ligaments (Fig. 3) [7, 19, 31].

The anatomic course and distribution of medial branch may feature considerable variability, thus complicating the punctual description of LFJs innervation [19]. Bogduk et al. [7] stated in their paper that medial branch provides innervation to LFJs of respective level and one level caudally with descending branches. This statement was in general validated from the vast majority of subsequently published papers (Fig. 3) $[11,19,29,31]$. There is, however, existing literature data supporting that LFJs innervation may be more complex, with emerging branches of sympathetic trunk and adjacent spinal ganglion participating as well $[19,25,34,35]$.

In their cadaveric study, Shuang et al. [31] concluded that innervation of a particular LFJs is provided by medial branches of the two adjacent DR. Medial branches of lumbar DR demonstrate a terminal descending portion, which may extent to 1-3 intervertebral levels. Medial branches of cranial DR tend to finally exhibit a shorter descending course in contrast to caudal. Furthermore, it was elucidated that articular branches from medial branch are also derived prior to entrance in fibro-osseous canal. These branches provide regional innervation to lateral and inferior portion of LFJs, whereas the terminally separated articular branches supply superior and medial aspects of LFJs [31].

L5 spinal segment features special anatomic characteristics in terms of neural distribution. First, DR presents a longer course than ventral ramus, rising at the excavation between the superior surface of sacral ala and the base of S1 SAP $[19,26]$. Perolat et al. [26] reviewed existing literature on FJ syndrome, quoting alongside data about LFJs anatomy. It was stated that L5 DR is bifurcated into a medial and an intermediate branch, with no presence of lateral branch [26]. However, this thesis is not universally accepted [31]. Medial branch of L5 DR subsequently runs caudally, giving rise to communicating branches to S1 DR [26].

\section{CONCLUSIONS}

Existing literature evidence indicates that LFJs represent complex structures in terms of anatomy, biomechanical sententiousness and functional importance. Despite the emerging interest on describing LFJs anatomy, a precise and universally accepted description of LFJs innervation remains absent. Future anatomic and radiologic studies with greater size of specimens may clarify this issue, contributing thus to better comprehension and optimisation of particular interventional procedures as LFJs denervation.

\section{Acknowledgements}

We desire to thank Mrs. Michaela Karamitsou for her significant contribution to graphic illustration of our paper.

Conflict of interest: None declared 


\section{REFERENCES}

1. Adams MA, Hutton WC. The mechanical function of the lumbar apophyseal joints. Spine (Phila Pa 1976). 1983; 8(3): 327-330, doi: 10.1097/00007632-19830400000017, indexed in Pubmed: 6623200.

2. Ahmed AM, Duncan NA, Burke DL. The effect of facet geometry on the axial torque-rotation response of lumbar motion segments. Spine (Phila Pa 1976). 1990; 15(5): 391-401, doi: 10.1097/00007632-199005000-00010, indexed in Pubmed: 2363067.

3. Alonso F, Kirkpatrick CM, Jeong W, et al. Lumbar facet tropism: a comprehensive review. World Neurosurg. 2017; 102: 91-96, doi: 10.1016/j.wneu.2017.02.114, indexed in Pubmed: 28279769.

4. An SJ, Seo MiS, Choi SIl, et al. Facet joint hypertrophy is a misnomer: A retrospective study. Medicine (Baltimore). 2018; 97(24): e11090, doi: 10.1097/ MD.0000000000011090, indexed in Pubmed: 29901623.

5. Ban $E$, Zhang $S$, Zarei $V$, et al. Collagen organization in facet capsular ligaments varies with spinal region and with ligament deformation. J Biomech Eng. 2017; 139(7), doi: 10.1115/1.4036019, indexed in Pubmed: 28241270.

6. Bogduk N. Clinical anatomy of the lumbar spine and sacrum. 3rd ed. Churchill Livingstone, Edinburgh 1997.

7. Bogduk N, Wilson AS, Tynan W. The human lumbar dorsal rami. J Anat. 1982; 134(Pt 2): 383-397, indexed in Pubmed: 7076562.

8. Chadha M, Sharma G, Arora SS, et al. Association of facet tropism with lumbar disc herniation. Eur Spine J. 2013; 22(5): 1045-1052, doi: 10.1007/s00586-012-2612-5, indexed in Pubmed: 23242621.

9. Cohen SP, Raja SN. Pathogenesis, diagnosis, and treatment of lumbar zygapophysial (facet) joint pain. Anesthesiology. 2007; 106(3): 591-614, doi: 10.1097/00000542200703000-00024, indexed in Pubmed: 17325518.

10. Demir-Deviren S, Singh S, Hanelin J. Superior recess access of the lumbar facet joint. Clin Spine Surg. 2017; 30(3): E169-E172, doi: 10.1097/BSD.0000000000000271, indexed in Pubmed: 28323695.

11. Demondion X, Vidal C, Glaude E, et al. The posterior lumbar ramus: $\mathrm{CT}$-anatomic correlation and propositions of new sites of infiltration. AJNR Am J Neuroradiol. 2005; 26(4): 706-710, indexed in Pubmed: 15814909.

12. Dupont G, Yilmaz E, Iwanaga J, et al. Ossification of the mamillo-accessory ligament: a review of the literature and clinical considerations. Anat Cell Biol. 2019; 52(2): 115-119, doi: 10.5115/acb.2019.52.2.115, indexed in Pubmed: 31338226.

13. Farfan HF, Sullivan JD. The relation of facet orientation to intervertebral disc failure. Can J Surg. 1967; 10(2): 179-185, indexed in Pubmed: 4225346.

14. Gao T, Lai Qi, Zhou S, et al. Correlation between facet tropism and lumbar degenerative disease: a retrospective analysis. BMC Musculoskelet Disord. 2017; 18(1): 483, doi: 10.1186/s12891-017-1849-x, indexed in Pubmed: 29166933.

15. Gellhorn AC, Katz JN, Suri P. Osteoarthritis of the spine: the facet joints. Nat Rev Rheumatol. 2013; 9(4): 216-224, doi: 10.1038/nrrheum.2012.199, indexed in Pubmed: 23147891.
16. Inoue N, Orías AA, Segami K. Biomechanics of the lumbar facet joint. Spine Surg Relat Res. 2020; 4(1): 1-7, doi: 10.22603/ssrr.2019-0017, indexed in Pubmed: 32039290.

17. Ishihara $H$, Matsui $H$, Osada $R$, et al. Facet joint asymmetry as a radiologic feature of lumbar intervertebral disc herniation in children and adolescents. Spine (Phila Pa 1976). 1997; 22(17): 2001-2004, doi: 10.1097/00007632199709010-00012, indexed in Pubmed: 9306530.

18. Jiang X, Chen D, Li Z, et al. Correlation between lumbar spine facet joint orientation and intervertebral disk degeneration: a positional MRI analysis. J Neurol Surg A Cent Eur Neurosurg. 2019; 80(4): 255-261, doi: 10.1055/s-00391683450, indexed in Pubmed: 30934096.

19. Kozera K, Ciszek B. Posterior branches of lumbar spinal nerves - part I: anatomy and functional importance. Ortop Traumatol Rehabil. 2016; 18(1): 1-10, doi: 10.5604/15093492.1198827, indexed in Pubmed: 27053304.

20. Kozera K, Ciszek B, Szaro P. Posterior branches of lumbar spinal nerves. Part II: lumbar facet syndrome - pathomechanism, symptomatology and diagnostic workup. Ortop Traumatol Rehabil. 2017; 19(2): 101-109, doi: 10.5604/15093492.1237716, indexed in Pubmed: 28508761 .

21. Louis R. Spinal stability as defined by the three-column spine concept. Anat Clin. 1985; 7(1): 33-42, doi: 10.1007/ BF01654627, indexed in Pubmed: 3994851.

22. Mahato NK. Facet dimensions, orientation, and symmetry at L5-S1 junction in lumbosacral transitional States. Spine (Phila Pa 1976). 2011; 36(9): E569-E573, doi: 10.1097/ BRS.0b013e3181f6ecb2, indexed in Pubmed: 21245783.

23. Mohanty SP, Pai Kanhangad M, Kamath S, et al. Morphometric study of the orientation of lumbar zygapophyseal joints in a South Indian population. J Orthop Surg (Hong Kong). 2017; 25(3): 2309499017739483, doi: 10.1177/2309499017739483, indexed in Pubmed: 29121821.

24. O'Leary SA, Link JM, Klineberg EO, et al. Characterization of facet joint cartilage properties in the human and interspecies comparisons. Acta Biomater. 2017; 54: 367-376, doi: 10.1016/j.actbio.2017.03.017, indexed in Pubmed: 28300721.

25. Pedersen H, Blunck C, Gardner E. The anatomy of lumbosacral posterior rami and meningeal branches of spinal nerves (sinu-vertebral nerves). J Bone Joint Surg. 1956; 38(2): 377-391, doi: 10.2106/00004623-19563802000015.

26. Perolat R, Kastler A, Nicot B, et al. Facet joint syndrome: from diagnosis to interventional management. Insights Imaging. 2018; 9(5): 773-789, doi: 10.1007/s13244-0180638-x, indexed in Pubmed: 30090998.

27. Proietti L, Schirò GR, Sessa S, et al. The impact of sagittal balance on low back pain in patients treated with zygoapophysial facet joint injection. Eur Spine J. 2014; 23 Suppl 6: 628-633, doi: 10.1007/s00586-014-3559-5, indexed in Pubmed: 25212449.

28. Putz R. The functional morphology of the superior articular processes of the lumbar vertebrae. J Anat. 1985; 143: 181-187, indexed in Pubmed: 3870725.

29. Saito T, Steinke H, Miyaki T, et al. The medial branch of the lateral branch of the posterior ramus of the spinal nerve. 
Surg Radiol Anat. 2006; 28(3): 228-234, doi: 10.1007/ s00276-006-0090-3, indexed in Pubmed: 16612554.

30. Saito T, Yoshimoto M, Yamamoto Y, et al. The medial branch of the lateral branch of the posterior ramus of the spinal nerve. Surg Radiol Anat. 2006; 28(3): 228-234, doi: 10.1007/ s00276-006-0090-3, indexed in Pubmed: 16612554.

31. Shuang F, Hou SX, Zhu JL, et al. Clinical anatomy and measurement of the medial branch of the spinal dorsal ramus. Medicine (Baltimore). 2015; 94(52): e2367, doi: 10.1097/ MD.0000000000002367, indexed in Pubmed: 26717379.

32. Song Q, Liu X, Chen DJ, et al. Evaluation of MRI and CT parameters to analyze the correlation between disc and facet joint degeneration in the lumbar three-joint complex. Medicine (Baltimore). 2019; 98(40): e17336, doi: 10.1097/ MD.0000000000017336, indexed in Pubmed: 31577728.

33. Steinke $H$, Saito T, Miyaki T, et al. Anatomy of the human thoracolumbar Rami dorsales nervi spinalis. Ann Anat. 2009; 191(4): 408-416, doi: 10.1016/j.aanat.2009.04.002, indexed in Pubmed: 19570665.

34. Suseki K, Takahashi Y, Takahashi K, et al. Innervation of the lumbar facet joints. Origins and functions. Spine (Phila Pa 1976). 1997; 22(5): 477-485, doi: 10.1097/00007632199703010-00003, indexed in Pubmed: 9076878.

35. Tessitore E, Molliqaj G, Schatlo B, et al. Clinical evaluation and surgical decision making for patients with lumbar discogenic pain and facet syndrome. Eur J Radiol. 2015; 84(5): 765-770, doi: 10.1016/j.ejrad.2014.03.016, indexed in Pubmed: 24801263.
36. Thorpe Lowis CG, Xu Z, Zhang M. Visualisation of facet joint recesses of the cadaveric spine: a micro-CT and sheet plastination study. BMJ Open Sport Exerc Med. 2018; 4(1): e000338, doi: 10.1136/bmjsem-2017-000338, indexed in Pubmed: 29527323.

37. Tulsi RS, Hermanis GM. A study of the angle of inclination and facet curvature of superior lumbar zygapophyseal facets. Spine (Phila Pa 1976). 1993; 18(10): 1311-1317, doi: 10.1097/00007632-199308000-00010, indexed in Pubmed: 8211363.

38. Twomey $\mathrm{L}$, Taylor J. Age changes in lumbar intervertebral discs. Acta Orthop Scand. 1985; 56(6): 496-499, doi: 10.3109/17453678508993043, indexed in Pubmed: 4090952.

39. Varlotta GP, Lefkowitz TR, Schweitzer M, et al. The lumbar facet joint: a review of current knowledge: part 1: anatomy, biomechanics, and grading. Skeletal Radiol. 2011; 40(1): 13-23, doi: 10.1007/s00256-010-0983-4, indexed in Pubmed: 20625896.

40. Wang H, Zhang Z, Zhou Y. Irregular alteration of facet orientation in lumbar segments: possible role in pathology of lumbar disc herniation in adolescents. World Neurosurg. 2016; 86: 321-327, doi: 10.1016/j.wneu.2015.09.029, indexed in Pubmed: 26409076.

41. Yang KH, King Al. Mechanism of facet load transmission as a hypothesis for low-back pain. Spine (Phila Pa 1976). 1984; 9(6): 557-565, doi: 10.1097/00007632-19840900000005, indexed in Pubmed: 6238423. 